\title{
Allocation of Cloudlets using Solution of Job Assignment Problem
}

\author{
Sonam Pathak, Manish Pandey, Kanu Geete
}

\begin{abstract}
Resource allocation policies play a key role in determining the performance of cloud. Service providers in cloud computing have to provide services to many users simultaneously. So the job of allocating cloudlets to appropriate virtual machines is becoming one of the challenging issues of cloud computing. Many algorithms have been proposed to allocate cloudlets to the virtual machines. Here in our paper, we have represented cloudlet allocation problem as job assignment problem and we have proposed Hungarian algorithm based solution for allocating cloudlets to virtual machines. The main objective is to minimize total execution time of cloudlets. Proposed algorithm is implemented in Cloudsim-3.03 simulator. We have done comparative analysis of the simulation results of proposed algorithm with the existing First Come First Serve (FCFS) scheduling policy and Min-Min scheduling algorithm. Proposed algorithm performs better than the above mentioned algorithms in terms of total execution time and makespan time (finishing time of last cloudlet).
\end{abstract}

Index Terms: CloudSim, cloudlets, Hungarian algorithm, resource allocation, virtual machines

\section{INTRODUCTION}

Cloud Computing is internet based computing in which services are provided on demand as per the requirements of clients. Three main services provided by cloud computing is infrastructure as a service (IAAS), platform as a service (PAAS) and software as a service (SAAS) [1]. Cloud computing is based on pay per usage model. Customers will have to pay only for what they consume. Cloud computing provide mobility, scalability and flexibility in service provisioning. In cloud, users' requests for resources are termed as cloudlets. These cloudlets need to be handled effectively with available resources. So main concern in cloud computing is to use efficient allocation algorithms in order to allocate virtual machines (VM) to the cloudlets [2].

Cloud computing environments are used for implementing scheduling algorithms. Some basic entities of cloud computing environments are Datacenter, Host, Virtual machines (VM), Tasks/Cloudlets, Datacenter Broker and Cloud Information Service (CIS) [3]. Fig 1 shows the pictorial representation for cloudlet allocation in cloud. Datacenter is group of different host. Each host may consist of different virtual machines of different specifications. CIS stores metadata of different cloud entities. CIS is kind of

Revised Manuscript Received on July 10, 2019.

Sonam Pathak, Computer Science and Engineering, Maulana Azad National Institute of Technology, Bhopal, India.

Manish Pandey, Computer Science and Engineering, Maulana Azad National Institute of Technology, Bhopal, India.

Kanu Geete, Computer Science and Engineering, Maulana Azad National Institute of Technology, Bhopal, India. repository in cloud computing environment. Datacenter broker works between clients and. cloud service supplier. It gathers all the required information related to availability of resources, usage of resources and cost of communication. Then according to the used scheduling algorithm datacenter broker allocates cloudlets to virtual machines.

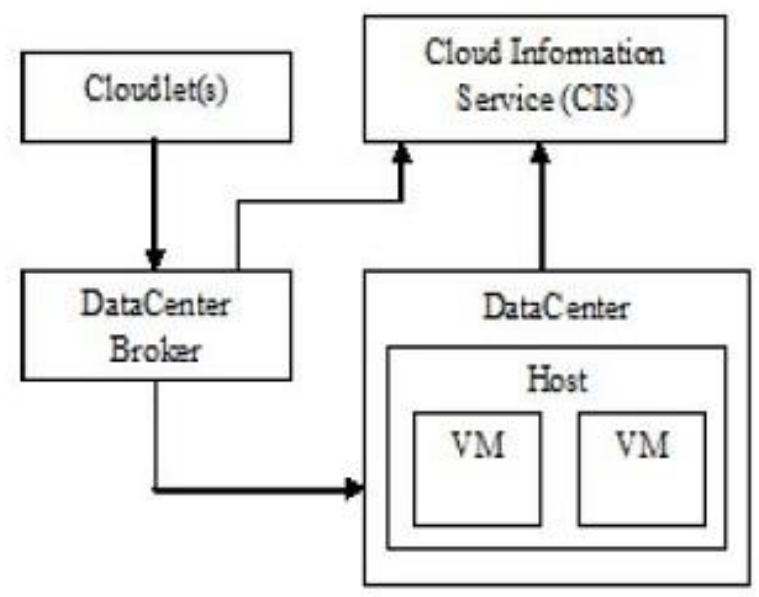

Fig.1. Cloudlet Allocation in Cloud Computing

In our paper, we have used one of the solutions of job assignment problem i.e. Hungarian algorithm for allocation of cloudlets. Our proposed algorithm minimizes total execution time of all cloudlets.

\section{RELATED WORK}

Cloudlet allocation is one of the most trending research topic in cloud computing. Several algorithms for cloudlet allocation have been proposed. The default policy used by Cloudsim toolkit is first come first serve policy (FCFS) [4]. FCFS simply allocates first cloudlet to first virtual machine, second cloudlet to second virtual machine and so on. Disadvantage of FCFS is that it does not consider which virtual machine will take less time for execution of particular cloudlet. Min-Min scheduling algorithm firstly computes minimum time required for execution of each cloudlet. Among all the cloudlets it selects the cloudlet with least execution time. Then it assigns this cloudlet to the virtual machine that computes minimum time for that cloudlet. This process continues for all cloudlet. [5] [6].

In Max-Min scheduling algorithm first step is same as Min-Min i.e. we calculate 
minimum execution time of each cloudlet. Then instead of selecting the cloudlet with least execution time we select the cloudlet with maximum execution time and repeat the process until last cloudlet is allocated [7]. A reliability based resource allocation has also been proposed by A B M Bodrul Alam and Mohammad Zulkernine [8]. The main goal of this allocation method is to

increase the reliability of cloud by increasing the reliability of its resources. These days, a lot of other domains including machine learning and big data utilize the capabilities of each other to give large efficient enterprise solutions in cloud. An extension of the shortest job first algorithm has been proposed by Mokhtar A. Alworafi [9]. This modified shortest job first algorithm performs better than the shortest job first algorithm by considering mean of all the cloudlets.

\section{Proposed Method}

We have related "Cloudlet Allocation Problem Model" with "Job Assignment Problem Model" [9]. We have proposed a linear programming model for mapping cloudlets to the virtual machines. Let us consider a set of virtual machines with $\mathrm{n}$ virtual machines and a set of cloudlets with $\mathrm{m}$ cloudlets. Assume that allocation is such that per virtual machine one cloudlet is allocated.

Let us take $n=m$. We will calculate the cost matrix $C=\left[c_{i j}\right]$ by using formula which is mentioned in (1). Here $C$ is an $n \times n$ cost matrix and $c_{i j}$ denotes $i, j$ entry of matrix.

$$
c_{i j}=l_{j} / v m_{i}
$$

$c_{i j}=$ Execution time of $\mathrm{j}^{\text {th }}$ cloudlet on $\mathrm{i}^{\text {th }}$ virtual machine

$\iota_{j}=$ length of $\mathrm{j}^{\text {th }}$ cloudlet in million instructions

$v m_{i}=$ capacity of $i^{\text {th }}$ virtual machine in million instructions per second

Let $Y=\left[y_{i j}\right]$ is another $n \times n$ matrix where $y_{i j}$ is $=\left\{\begin{array}{l}1, \text { if cloud let } j \text { is allocated to virtual machine } i \\ 0, \text { if cloudet } j \text { is not allocated to virtual machine } i\end{array}\right.$

Our goal is to optimize the cost $q(Y)$. Here cost is the total time required for execution of all cloudlets. Linear model of our cloudlet allocation problem is as follows:

$$
\text { Minimize } q(Y)=\sum_{j=1}^{+n} \sum_{i=1}^{\dagger} c_{i j} y_{i j}
$$

Subjects to

$$
\begin{array}{lr}
\sum_{i=1}^{n} y_{i j}=1 & \text { for } \mathrm{j}=1,2,3 \ldots \mathrm{m} \\
\sum_{j=1}^{n+n} y_{i j}=1 & \text { for } \mathrm{i}=1,2,3 \ldots \ldots \mathrm{n} \\
y_{i j}= & 0 \text { or } 1
\end{array}
$$

This linear programming model of cloudlet allocation is same as job assignment model. So we can use the solution of assignment problem to solve our cloudlet allocation problem. We have used Hungarian Method based solution for our problem. Hungarian Method is one of the standard solutions of job assignment problem which we are using for solving our cloudlet allocation problem. The objective of proposed method is to lessen the overall execution time of all cloudlets.
In the proposed method, we have considered equal number of cloudlets and equal number of virtual machines. If available virtual machines and cloudlets are not equal in number then we add dummy rows. We assume that each cloudlet needs to be allocated to just single VM and each VM will execute just one cloudlet.

\section{A. Method Description}

Let us take an example with 3 virtual machines and 3 cloudlets.

Table1. MIPS Value for Virtual Machines

\begin{tabular}{|l|c|c|c|}
\hline Parameter & VM1 & VM2 & VM3 \\
\hline Capacity & 200 & 500 & 100 \\
\hline
\end{tabular}

Table2. Length of Cloudlets

\begin{tabular}{|l|c|c|c|}
\hline Parameter & CL1 & CL2 & CL3 \\
\hline Length & 20000 & 60000 & 40000 \\
\hline
\end{tabular}

Step1: Compute the initial cost matrix by the mentioned formula.

Table3. Cost Matrix

\begin{tabular}{|l|l|l|l|}
\hline & CL1 & CL2 & CL3 \\
\hline VM1 & 100 & 300 & 200 \\
\hline VM2 & 40 & 120 & 80 \\
\hline VM3 & 200 & 400 & 600 \\
\hline
\end{tabular}

Step2: Subtracting minimum element of each row from all the values of its row.

Table4. Cost Matrix after performing step2

\begin{tabular}{|c|c|c|c|}
\hline & CL1 & CL2 & CL3 \\
\hline VM1 & 0 & 200 & 100 \\
\hline VM2 & 0 & 80 & 40 \\
\hline VM3 & 0 & 200 & 400 \\
\hline
\end{tabular}

Step3: Subtracting minimum element of each column from all the elements of its column.

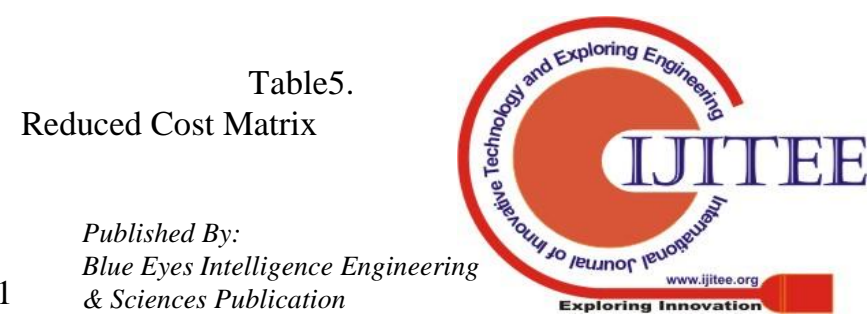




\begin{tabular}{|c|c|c|c|}
\hline & CL1 & CL2 & CL3 \\
\hline VM1 & 0 & 120 & 60 \\
\hline VM2 & 0 & 0 & 0 \\
\hline VM3 & 0 & 120 & 360 \\
\hline
\end{tabular}

Step4: Now we find out minimum number of lines required to cover all zeroes in Reduced Cost Matrix. As we can see two lines will be needed one at column 1 and one at row 2 .

If number of lines is same as number of available virtual machines then we do mapping of cloudlet and virtual machine. If number of lines is less then number of virtual machine then we find out minimum value element among all uncovered elements. We subtract this least value element from all the uncovered elements and add this element to the elements where lines intersect with each other.

Table6. Final Cost Matrix

\begin{tabular}{|l|l|l|l|}
\hline & CL1 & CL2 & CL3 \\
\hline VM1 & 0 & 60 & 0 \\
\hline VM2 & 60 & 0 & 0 \\
\hline VM3 & 0 & 60 & 300 \\
\hline
\end{tabular}

Step5: Now we require three lines to cover all zero, one line at column 1, one line at row 2 and the last one at column 3 . We do mapping of cloudlet to virtual machine based on zeros.

VM1-> CL3

VM2-> CL2

VM3-> CL1

Now we get total execution time (Cost) by adding the value of corresponding mapping in initial cost matrix.

Cost $=200+120+200=520$ seconds

\section{B. Algorithm}

\section{Input:}

$\mathrm{n}$ : available virtual machines

$\mathrm{m}$ : available cloudlets

\section{Output:}

Cost: Total execution time of all cloudlets

$1: / *$ Initialize value of cost matrix */

2: for $\mathrm{i}=1$ to $\mathrm{n}$

3: for $\mathrm{j}=1$ to $\mathrm{m}$

4: $\quad \mathrm{c}_{\mathrm{ij}}=\mathrm{l}_{\mathrm{j}} / \mathrm{vm}_{\mathrm{i}}$

5: end for

6: end for

7: if $\mathrm{n}$ is not equal to $\mathrm{m}$

8: put dummy value in matrix to convert matrix in Square matrix

\section{9: end if}

10: $\operatorname{minr}_{i}=$ smallest element in row $\mathrm{i}$

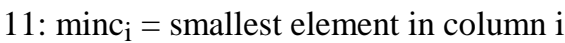

12: /* calculate reduced cost matrix */

13: for $\mathrm{i}=1$ to $\mathrm{n}$

14: for $\mathrm{j}=1$ to $\mathrm{m}$

15: $\quad c_{i j}=c_{i j}-\operatorname{minr}_{i}$

16: end for

17: end for

18: for $i=1$ to $n$

19: for $\mathrm{j}=1$ to $\mathrm{m}$

20: $\quad c_{j i}=c_{j i}-\operatorname{minc}_{\mathrm{i}}$

21: end for

22: end for

23: /* calculate final cost matrix */

24: $\mathrm{k}=$ min_no_line ()

25 : if $\mathrm{k}<\mathrm{n}$

27: for $i=1$ to $n$

28: for $\mathrm{j}=1$ to $\mathrm{m}$

29: if $\mathrm{c}_{\mathrm{ij}}$ is not covered by any of line

30: $\quad c_{i j}=c_{i j}-$ minimum of all uncovered elements

31: else if $c_{i j}$ is covered by two line

32: $\quad \mathrm{c}_{\mathrm{ij}}=\mathrm{c}_{\mathrm{ij}}+$ minimum of all uncovered elements

33: end if

34: end for

35: end for

36: end if

37: /* Finding mapping of cloudlets to virtual machines */

38: Find_mapping() for all cloudlets

39: adding their execution time will give total execution time i.e. Cost

40: return Cost

\section{EXPERIMENTAL RESULTS AND COMPARATIVE} ANALYSIS

We have used open source simulator named CloudSim-3.0.3 and Eclipse Java IDE for our experimental purpose. Cloudsim toolkit gives the user the flexibility to implement their own algorithms for provisioning of resources [11][12]. 


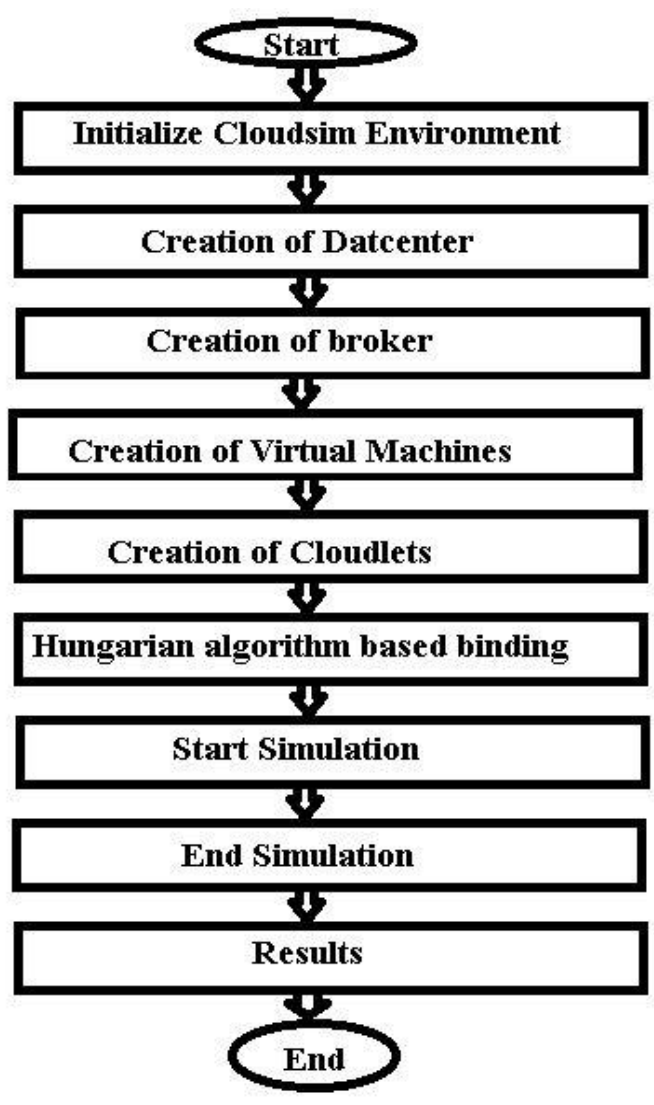

Fig.2. Cloudsim Lifecycle

Cloudsim gives classes for virtual machines, data centers, applications, clients and scheduling methods. Different stages of Cloudsim lifecycle is shown in Fig.2. Cloudsim life cycle starts with initialization of Cloudsim environment and ends with simulation results [13][14][15]. Scheduling algorithm is applied after creation of cloudlets. We have executed our proposed algorithm in Cloudsim and compared its result with conventional FCFS policy and Min-Min scheduling algorithm. Simulation results are given in tables 9, 10 and 11 .

Table7. Length of Cloudlets for 3 different jobs

\begin{tabular}{|c|c|l|l|}
\hline \multirow{2}{*}{ Cloudlet Id } & Job1 & Job2 & Job3 \\
\cline { 2 - 4 } & (Length) & (Length) & (length) \\
\hline 0 & 20000 & 200000 & 40000 \\
\hline 1 & 60000 & 20000 & 120000 \\
\hline 2 & & & \\
\hline 3 & 90000 & 80000 & 60000 \\
\hline 4 & 40000 & 90000 & 20000 \\
\hline & & & 70000 \\
\hline
\end{tabular}

Table8. MIPS of Virtual Machines

\begin{tabular}{|c|c|c|c|c|c|}
\hline VM ID & 0 & 1 & 2 & 3 & 4 \\
\hline MIPS & 1000 & 500 & 200 & 2000 & 250 \\
\hline
\end{tabular}

Table9. Result of Conventional FCFS Scheduling in Cloudsim

\begin{tabular}{|c|c|c|c|c|c|c|}
\hline \multirow{2}{*}{$\begin{array}{c}\text { Cloudlet } \\
\text { id }\end{array}$} & \multicolumn{2}{|c|}{ Job1 } & \multicolumn{2}{c|}{ Job2 } & \multicolumn{2}{c|}{ Job3 } \\
\cline { 2 - 7 } & $\begin{array}{c}\text { Exec. } \\
\text { Time }\end{array}$ & $\begin{array}{c}\text { VM } \\
\text { id }\end{array}$ & $\begin{array}{c}\text { Exec. } \\
\text { Time }\end{array}$ & $\begin{array}{c}\text { VM } \\
\text { ID }\end{array}$ & $\begin{array}{c}\text { Exec. } \\
\text { Time }\end{array}$ \\
\hline 0 & 0 & 20 & 0 & 200 & 0 & 40 \\
\hline 1 & 1 & 120 & 1 & 40 & 1 & 240 \\
\hline 2 & 2 & 450 & 2 & 400 & 2 & 300 \\
\hline 3 & 3 & 20 & 3 & 45 & 3 & 10 \\
\hline 4 & 4 & 480 & 4 & 40 & 4 & 280 \\
\hline $\begin{array}{c}\text { Total } \\
\text { Exec. } \\
\text { Time }\end{array}$ & 1090 sec & $725 \mathrm{sec}$ & $870 \mathrm{sec}$ \\
\hline
\end{tabular}

Table10. Result of Min-Min scheduling in Cloudsim

\begin{tabular}{|c|c|c|c|c|c|c|}
\hline \multirow{2}{*}{$\begin{array}{c}\text { Cloudlet } \\
\text { id }\end{array}$} & \multicolumn{2}{|c|}{ Job1 } & \multicolumn{2}{c|}{ Job2 } & \multicolumn{2}{c|}{ Job3 } \\
\cline { 2 - 7 } id & $\begin{array}{c}\text { Exec. } \\
\text { Time }\end{array}$ & $\begin{array}{c}\text { VM } \\
\text { id }\end{array}$ & $\begin{array}{c}\text { Exec. } \\
\text { Time }\end{array}$ & $\begin{array}{c}\text { VM } \\
\text { id }\end{array}$ & $\begin{array}{c}\text { Exec. } \\
\text { Time }\end{array}$ \\
\hline 0 & 0 & 20 & 1 & 20 & 0 & 20 \\
\hline 1 & 1 & 120 & 2 & 160 & 2 & 180 \\
\hline 2 & 4 & 600 & 0 & 1000 & 1 & 300 \\
\hline 3 & 3 & 20 & 4 & 5 & 3 & 20 \\
\hline 4 & 2 & 360 & 3 & 360 & 4 & 480 \\
\hline $\begin{array}{c}\text { Total } \\
\text { Exec. } \\
\text { Time }\end{array}$ & $1120 \mathrm{sec}$ & $1545 \mathrm{sec}$ & $1000 \mathrm{sec}$ \\
\hline
\end{tabular}


Table11. Result of our proposed Hungarian Scheduling in

\begin{tabular}{|c|c|c|c|c|c|c|}
\hline \multirow{2}{*}{$\begin{array}{c}\text { Cloudlet } \\
\text { id }\end{array}$} & \multicolumn{2}{|c|}{ Job1 } & \multicolumn{2}{c|}{ Job2 } & \multicolumn{2}{c|}{ Job3 } \\
\cline { 2 - 7 } id & $\begin{array}{c}\text { Exec. } \\
\text { Time }\end{array}$ & $\begin{array}{c}\text { VM } \\
\text { id }\end{array}$ & $\begin{array}{c}\text { Exec. } \\
\text { Time }\end{array}$ & $\begin{array}{c}\text { VM } \\
\text { id }\end{array}$ & $\begin{array}{c}\text { Exec. } \\
\text { Time }\end{array}$ \\
\hline 0 & 2 & 90 & 3 & 90 & 4 & 70 \\
\hline 1 & 1 & 120 & 2 & 160 & 2 & 120 \\
\hline 2 & 0 & 100 & 4 & 50 & 3 & 100 \\
\hline 3 & 4 & 60 & 0 & 100 & 1 & 60 \\
\hline 4 & 3 & 160 & 1 & 80 & 0 & 160 \\
\hline $\begin{array}{c}\text { Total } \\
\text { Exec. } \\
\text { Time }\end{array}$ & $530 \mathrm{sec}$ & 480 sec & 510 sec \\
\hline
\end{tabular}

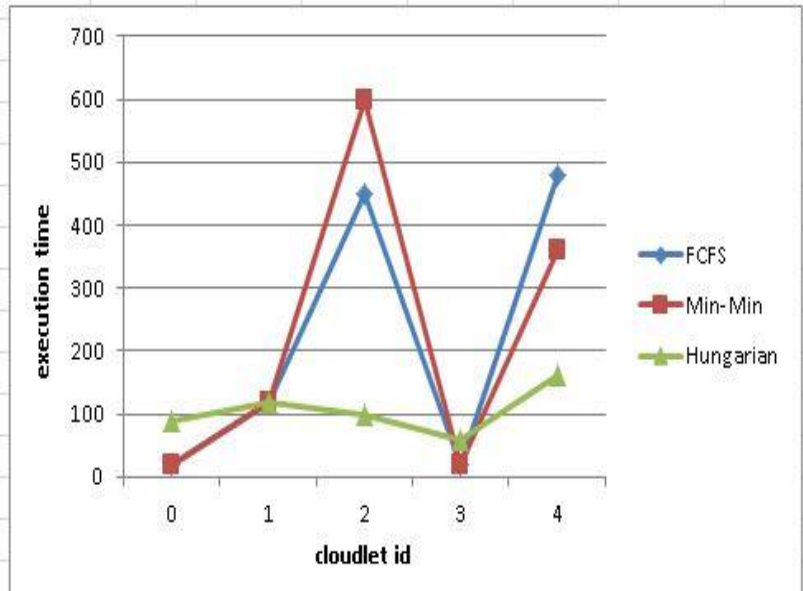

Fig.3. Execution Time of Cloudlets for job1

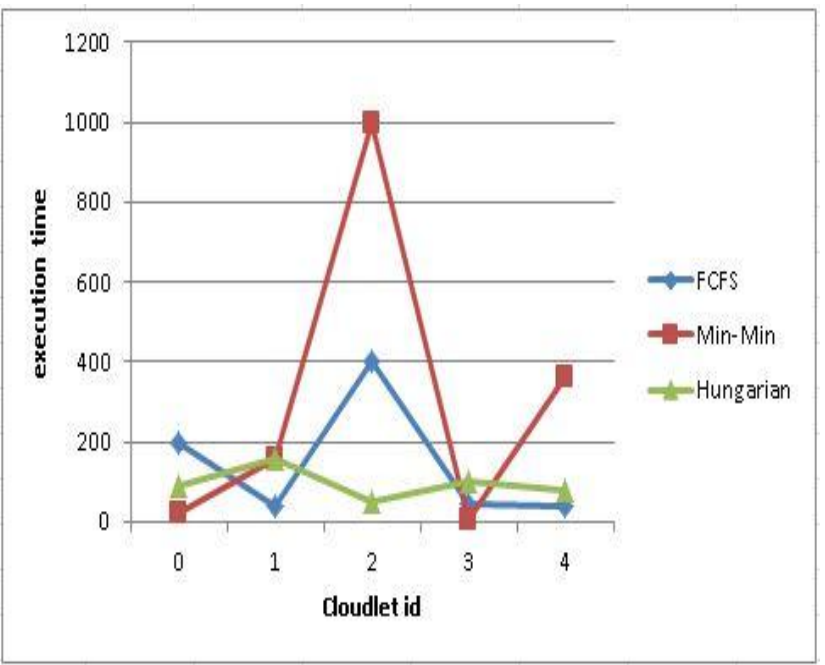

Fig.4. Execution Time of Cloudlets for job2

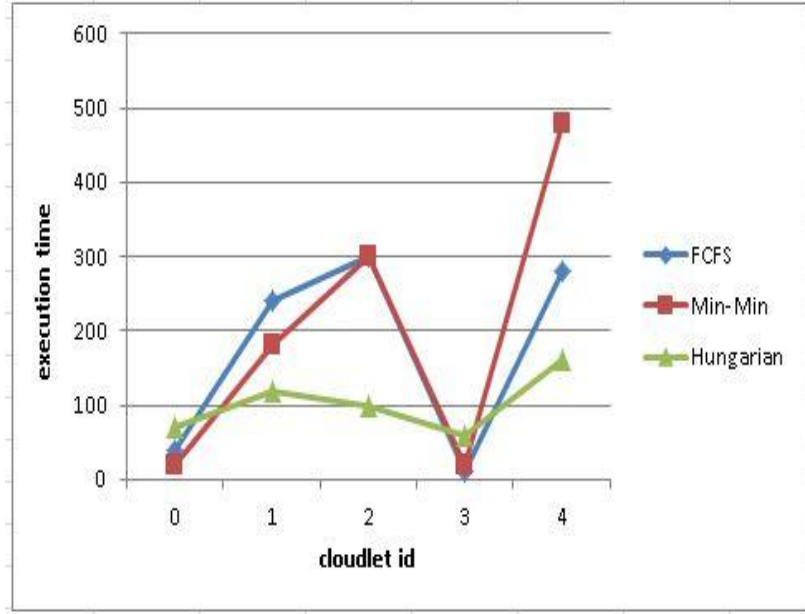

Fig.5. Execution Time of Cloudlets for job3

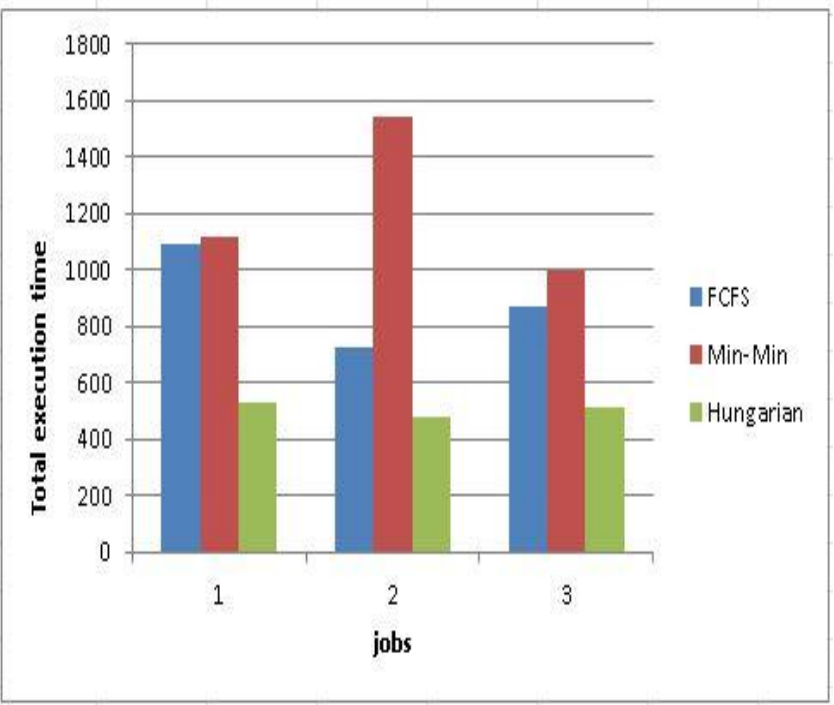

Fig.6. Comparing total execution time of proposed algorithm with FCFS and Min-Min scheduling algorithm for 3 different jobs

Fig. 3, 4 and 5 shows graphs of execution time for job1, job2 and job3 respectively. We have shown comparative analysis of total execution time of each job in Fig.6. Table 12 shows makespan time for 3 jobs using proposed method, conventional policy and min-min scheduling. Fig.7 gives graphical representation for comparing makespan time. We can clearly see from the tables and graphs that total execution time and makespan time is improved using our proposed method. Rate of improvement in makespan time for job1 using our proposed method is $66.67 \%$ over FCFS and $73.33 \%$ over Min-Min, for job2 improvement is $60 \%$ over FCFS and $84 \%$ over Min-Min and for job3 improvement is $37.5 \%$ over FCFS and $66.67 \%$ over Min-Min algorithm. 
Table12. Comparing Makespan time for job1, job2 and job3 using FCFS, Min-Min and Proposed approach

\begin{tabular}{|c|c|c|c|}
\hline Job & FCFS & Min-Min & Proposed Method \\
\hline 1 & 480 & 600 & 160 \\
\hline 2 & 400 & 1000 & 160 \\
\hline 3 & 300 & 480 & 160 \\
\hline
\end{tabular}

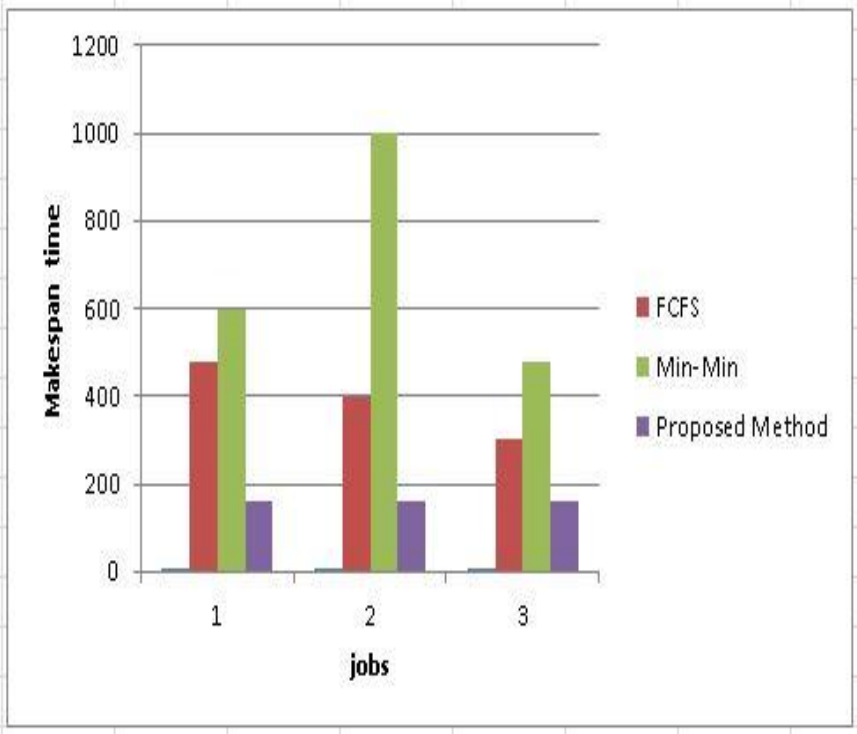

Fig.7. Comparing Makespan time of proposed method with existing ones

\section{CONCLUSION AND FUTURE WORK}

In this paper, we have proposed Hungarian Algorithm based solution for cloudlet allocation problem by keeping in mind the issues of resource allocation problem. Our proposed algorithm allocates each cloudlet to appropriate virtual machine in such a way that overall execution time is minimized. Consequently makespan time is also minimized. We have implemented our proposed algorithm, first come first serve scheduling (FCFS) and Min-Min scheduling algorithm in Cloudsim tool and we have compared the simulation results of these three algorithms. Our proposed algorithm is more efficient than the existing conventional FCFS binding policy and Min-Min scheduling algorithm in terms of total execution time and makespan time.

In future, our proposed method may be used for allocation of cloudlets in real time. By keeping in mind the efficiency of the proposed approach, same method can be used for fog computing and mobile computing applications.

\section{REFERENCES}

1. Saurabh Kumar Garg, Steeve Versteeg and Rajkumar Buyya, "A Framework for ranking of cloud computing services", S.K. Garg et al. / Future Generation Computer Systems 29 (2013) 1012-1023.

2. MR.Manan D. Shah, MR.Amit A. Kariyani and MR.Dipak L. Agrawal, "Allocation of Virtual Machines In Cloud ComputingUsing Load Balancing Algorithm" IRACST - International Journal of Computer Science and Information Technology \& Security (IJCSITS), ISSN: 2249-9555 Vol. 3, No.1, February 2013.

3. Tarun Goyal, Ajit Singh and Akansha Goyal, "Cloudsim:Simulator for cloud computing infrastructure and modeling" International Conference on Modeling, optimization and computing (ICMOC-2012).

4. Dr. Amit Agarwal and Saloni Jain, "Efficient Optimal Algorithm of Task Scheduling in Cloud Computing Environment" International Journal of Computer Trends and Technology (IJCTT) - volume 9.

5. Gaurang Patel, Rutvik Mehta and Upendra Bhoi, "Enhanced Load Balanced Min-Min Algorithm for Static Meta Task Scheduling in Cloud Computing", $3^{\text {rd }}$ International Conference on Recent Trends In Computing 2015 (ICRTC-2015).

6. Neha Sharma and Dr. Sanjay Tyagi , "A Comparative Analysis of MinMin and Max-Min Algorithms based on the Makespan Parameter" International Journal of Advanced Research in Computer Science.

7. Upendra Bhoil and Purvi N. Ramanuj2, "Enhanced Max-min Task Scheduling Algorithm in Cloud Computing", Web Site: www.ijaiem.org Email: editor@ijaiem.org,editorijaiem@gmail.com Volume 2, Issue 4, April 2013.

8. A B M Bodrul Alam, Mohammad Zulkernine and Anwar Haque, "A Reliability-Based Resource Allocation Approach for Cloud Computing", 2017 IEEE 7th International Symposium on Cloud and Service Computing

9. Mokhtar A. Alworafi, Atyaf Dhari and Asma A. Al-Hashmi, "An Improved SJF Scheduling Algorithm in Cloud Computing Environment" 2016 International Conference on Electrical, Electronics, Communication, Computer and Optimization Techniques (ICEECCOT).

10. "Linear Programming Applications-nptel" https://nptel.ac.in/courses/110106059/Module\%201/Lecture\%202.pdf

11. Gibet Tani Hicham and El Amrani Chaker, "Cloud Computing CPU Allocation and Scheduling Algorithms Using CloudSim Simulator" International Journal of Electrical and Computer Engineering IJECE)Vol. 6, No. 4, August 2016, pp. 1866 1879 ISSN: 2088.

12. Pradeep Singh Rawat, G. P. Saroha and Varun Barthwal"Quality of Service Evaluation of SaaS Modeler (Cloudlet) Running on Virtual Cloud Computing Environment using CloudSim"International Journal of Computer Applications (0975-8887) Volume 53-No.13, September 2012 "'.

13. Suchintan Mishra and Manmath Narayan Sahoo, "On using CloudSim as a Cloud Simulator: The Un-official Manual", Research · December 2017 DOI: 10.13140/RG.2.2.30215.91041

14. Arezoo Khatibi and Omid Khatibi, "Criteria for the CloudSim Environment", arXiv:1807.03103v1 [cs.DC] 14 Jun 2018

15. Ranjan Kumar and G. Sahoo, "Cloud Computing Simulation Using CloudSim", International Journal of Engineering Trends and Technology (IJETT) - Volume8 Number 2-Feb 2014

\section{AUTHORS PROFILE}

First Author: Sonam Pathak, Research Scholar in Computer Science and engineering at Maulana Azad National Institute of Technology, Bhopal Email-22pathaksona@gmail.com

Second Author : Dr Manish Pandey, Assistant Professor at Maulana Azad National Institute of Technology, Bhopal, India,.

Email- contactmanishpandey@yahoo.co.in

Third Author: Kanu Geete, Phd scholar at Maulana Azad National Institute of Technology, Bhopal, India.

Email-kanu.geete29@gmail.com 